\title{
A Conserved microRNA Signal Specifies Leaf Polarity
}

\author{
M.C.P. Timmermans, ${ }^{*}$ M.T. JuARez, ${ }^{* \dagger}$ AND T.L. PhelPS-DurR ${ }^{*}$ \\ "Cold Spring Harbor Laboratory, Cold Spring Harbor, New York 11724; ${ }^{\dagger}$ Graduate Program in Genetics, \\ Stony Brook University, Stony Brook, New York 11794
}

Plant shoots are characterized by indeterminate growth resulting from the action of a population of stem cells in the central zone of the shoot apical meristem (SAM) (Fig. 1). These stem cells give rise to peripheral derivatives from which lateral organs, such as leaves and flowers, arise. Leaves of higher plants exhibit a varying degree of asymmetry along their adaxial/abaxial (upper/lower) axis. This asymmetry is thought to reflect inherent positional differences in the developing organ relative to the SAM (Wardlaw 1949). The adaxial/dorsal side of the leaf develops in close proximity to the stem cells in the SAM, whereas the abaxial/ventral side develops at a greater distance from the apex. Evidence that the meristem is required for pattern formation within lateral organs came from early surgical experiments in potato (Sussex 1951, 1955). Incisions that separate the incipient primordium from the central zone of the SAM resulted in formation of a radially symmetric abaxialized leaf, suggesting that a signal from the SAM is required to specify adaxial cell fate. The exact nature of this adaxializing signal remains elusive despite the recent identification of several gene families required for the determination of adaxial or abaxial identity. These recent studies have, however, identified a microRNA (miRNA) signal that originates

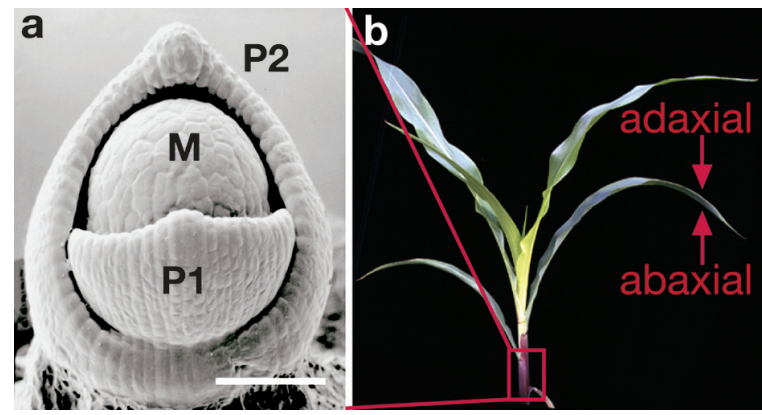

Figure 1. Leaf primordia arise on the flank of the shoot apical meristem (SAM) and become patterned along the adaxial/abaxial axis in response to signals from the SAM. (a) Scanning electron micrograph of a maize shoot apex. The meristem (M) contains a population of stem cells that allows the continued initiation of leaf primordia. The youngest leaf primordium is indicated as P1, the second youngest as P2, etc. (b) 14-day-old maize seedling. The red box marks the approximate position of the SAM within the surrounding older leaves that have distinct adaxial/upper and abaxial/lower surfaces. from below the incipient leaf and sets up the abaxial domain. Adaxial/abaxial leaf polarity may thus be established by two opposing signals that both originate outside the incipient primordium; the classical adaxializing signal from the tip of the SAM and the miRNA signal from a potential signaling center below the incipient leaf.

\section{ESTABLISHMENT OF POLARITY IN DEVELOPING LEAVES}

In Arabidopsis, PHABULOSA (PHB), PHAVOLUTA (PHV), and REVOLUTA (REV), members of the class III homeodomain-leucine zipper (HD-ZIPIII) family of proteins, promote adaxial identity in developing lateral organs (McConnell et al. 2001; Otsuga et al. 2001; Emery et al. 2003). By contrast, the KANADI (KAN) genes, which encode transcriptional regulators of the GARP family, are required for abaxial cell fate determination (Eshed et al. 2001, 2004; Kerstetter et al. 2001). Both the $H D$-ZIPIII and the KAN genes are expressed throughout the incipient leaf primordium, but shortly after the primordium emerges from the SAM, their expression becomes restricted to mutually exclusive domains on the adaxial and abaxial side of the developing organ, respectively. Once this adaxial/abaxial polarity is established, it is interpreted by other downstream genes that lead to the differentiation of adaxial and abaxial specific cell types and to mediolateral blade outgrowth at the adaxial/abaxial boundary (Waites and Hudson 1995). Among these are the $Y A B B Y$ genes, a third family of transcriptional regulators, that act at least in part downstream of the $H D$ ZIPIII and KAN genes and are required for abaxial cell fate and lamina expansion (Sawa et al. 1999; Siegfried et al. 1999; Eshed et al. 2001, 2004; Kumaran et al. 2002).

The mechanisms by which the HD-ZIPIII and KAN expression domains become restricted to opposing sides of the primordium upon its emergence from the SAM is unclear. Persistent uniform expression of KAN1 leads to the abaxialization of lateral organs (Eshed et al. 2001; Kerstetter et al. 2001). Thus, specification of adaxial identity requires the suppression of abaxial determinants. The phenotype resulting from loss of $P H B, P H V$, and $R E V$ function resembles the phenotype caused by ectopic $K A N$ expression, suggesting that the $H D-Z I P I I I$ genes act at least in part to spatially restrict the $K A N$ expression domain (Emery et al. 2003). HD-ZIPIII proteins contain a 
START lipid-sterol binding-like domain that is required for protein function (Otsuga et al. 2001). Therefore, PHB, $\mathrm{PHV}$, and REV may become activated upon interaction with a ligand, perhaps the meristem-borne signal proposed by the surgical experiments (McConnell et al. 2001; Juarez et al. 2004a). Such activation could direct the down-regulation of $K A N$ expression, thus suppressing abaxial identity and leading to the specification of adaxial cell fate. However, what suppresses the expression of $H D$-ZIPIII genes on the abaxial side? This spatial restriction of $P H B, P H V$, and $R E V$ expression is likely to involve an RNA-interference (RNAi)-like mechanism. Transcripts from all three genes contain a complementary site for miRNA 165 (miR165) and miR166, which can direct their cleavage in vitro (Reinhart et al. 2002; Rhoades et al. 2002; Tang et al. 2003; see below). Disruption of the miR165/166 complementary site, as in gain-of-function phb-d and phv-d alleles, prevents this miRNA-directed cleavage. Such mutations lead to ectopic abaxial expression of mutant transcripts and adaxialization of leaves and other lateral organs (McConnell et al. 2001). Similar dominant mutations in $R E V$ are less severe, but cause adaxial/abaxial patterning defects in the normally polarized vascular bundles (Emery et al. 2003; Zhong and Ye 2004).

\section{SPECIFICATION OF ADAXIAL/ABAXIAL POLARITY DURING MAIZE LEAF DEVELOPMENT}

Adaxial/abaxial asymmetry in maize leaves similarly depends on the polarized expression of members of the hd-zipIII gene family (Juarez et al. 2004a,b). rolled leafl ( $r l d 1$ ), which encodes a close homolog of REV, is normally expressed in the presumptive central zone of the SAM and in a stripe of cells from the center of the SAM to the site of leaf initiation (Fig. 2a,b). In the P1 primordium, rld 1 is expressed along the adaxial domain as well as in the midvein region. rldl expression persists during primordium development in the vasculature and on the adaxial side near the margins. The expression pattern of a maize $P H B$ homolog is comparable to that of $r l d l$ although the level of $p h b$ is relatively more abundant in the developing vasculature. These expression patterns resemble those of the HD-ZIPIII genes in Arabidopsis and are consistent with a conserved role for the maize $h d$ zipIII genes in meristem function and adaxial fate determination. However, the overlapping expression patterns of $r l d l$ and $p h b$ suggest these genes probably act redundantly in these processes.

The adaxial-specific expression of $r l d 1, p h b$, and potential other $h d$-zipIII family members is in part mediated by leafbladeless 1 (lbl1) (Juarez et al. 2004a; M. Juarez and M. Timmermans, unpubl.). Recessive mutations in lbll lead to the formation of abaxialized leaves. The most severely affected $l b l l$ mutant leaves are radially symmetric and resemble the abaxialized leaves that arise following the surgical separation of leaf initials from the central region of the SAM (Timmermans et al. 1998). The levels of $r l d l$ and $p h b$ transcripts are dramatically reduced or

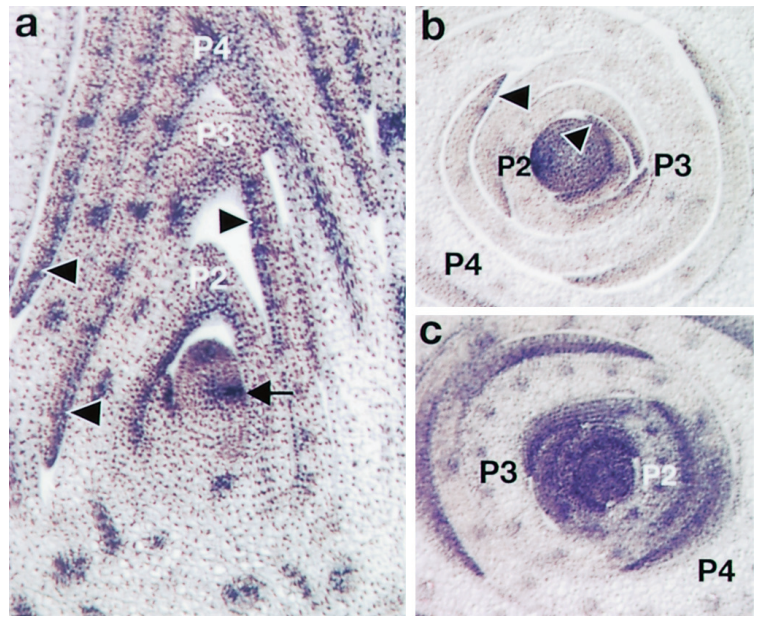

Figure 2. $r l d l$ expression is altered in $\operatorname{Rld} 1$ leaf primordia. $(a, b)$ Longitudinal $(a)$ and transverse $(b)$ sections through wild-type apices showing $r l d 1$ expression in the SAM, vasculature, and on the adaxial side of P1 and older leaf priomordia (arrowheads). The arrow marks rld 1 expression near the site of leaf initiation. (c) Transverse section through a Rldl mutant apex showing misexpression of rldl on the abaxial side of young leaf primordia. Leaf primordia P2-P4 are indicated. (Reprinted, with permission, from Juarez et al. 2004b [CNature Publishing Group; http://www.nature.com].)

lost in $l b l l$ leaves, suggesting that these genes act downstream of $l b l l$ in the pathway leading to adaxial identity.

Adaxial-specific expression of rld1 may also depend on the action of miR165 or miR166. The miR165/166 complementary site is conserved between rld1 and the Arabidopsis HD-ZIPIII genes. Moreover, four dominant mutant alleles of rldl were shown to result from single nucleotide substitutions in the $5^{\prime}$ end of the miR165/166 complementary site (Fig. 3a) (Juarez et al. 2004b). Such dominant Rldl mutations have no effect on the meristematic expression pattern of $r l d 1$, but lead to misexpression of rldl on the abaxial side of incipient and P1 leaf primordia (Fig. 2c). As in wild type, the rldl expression domain in older Rldl primordia becomes increasingly more confined to the nondetermined cells near the margins. However, the domain of strong rldl expression at the margins is broader and includes the abaxial side. Moreover, weak rldl expression persists on both the adaxial and abaxial site in the central region of $\mathrm{P} 2$ and older Rld1 primordia. These latter changes in $r l d 1$ expression are associated with an upward curling of the Rldl leaf blade caused by adaxialization or partial reversal of adaxial/abaxial leaf polarity (Fig. 3c) (Nelson et al. 2002; Juarez et al. 2004a). The ligule normally forms on the adaxial side of the leaf, and the wild-type adaxial epidermis has distinctive hairs and strengthening cells (Fig. 3b). Adaxial/abaxial polarity is also reflected in the patterning of the vasculature, in that xylem tissue differentiates toward the adaxial side whereas phloem forms on the abaxial side of the leaf. In Rld1 leaves, epidermal cell types are displaced from the adaxial to the abaxial leaf surface. In addition, adaxialized sectors often arise on either side of 
the midvein. Such sectors lack minor vascular bundles and their associated photosynthetic cell types and develop an ectopic ligule on the abaxial side of the leaf (Fig. 3d).

Characterization of the dominant Rld1, phb-d, phv-d and rev- $d$ alleles thus implicates an miRNA in the establishment of adaxial/abaxial polarity in both maize and Arabidopsis. The spatial regulation of hd-zipIII genes by miR165/166 may even date back to the mosses, which last shared a common ancestor with Arabidopsis and maize over 400 million years ago (Floyd and Bowman 2004). Other miRNAs with complementarity to developmentally important regulatory genes have been identified in plants as well as animals. The facts that miRNAs are present in such diverse species and control a broad range of targets imply an important role for this recently dis-

a
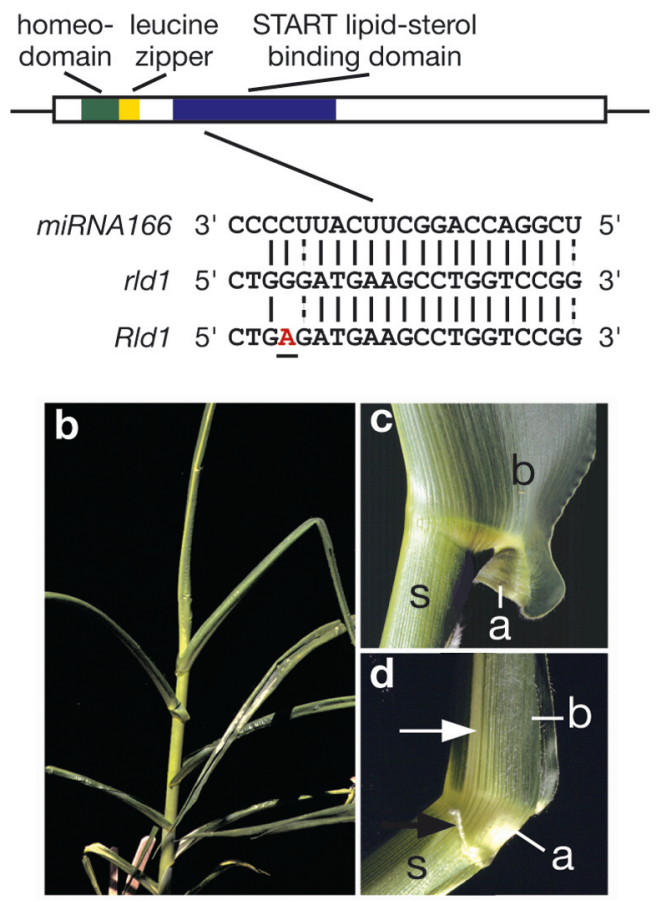

Figure 3. Dominant $R l d l$ alleles result from a point mutation in the miR166 complementary site and affect adaxial/abaxial polarity in the leaf. (a) Organization of RLD1, a member of the HD-ZIPIII family of proteins. rld 1 encodes an 840-amino-acid protein containing a homeodomain (green), leucine-zipper domain (yellow), and START lipid-sterol binding domain (blue). The approximate position of the miR166 complementary site and an alignment of miR166 with the target sites in rld1 and the mutant Rldl alleles are shown. Solid lines, Watson-Crick base pairs; dotted lines, RNA base pairs. The nucleotide substitution present in the four dominant Rld1 alleles is highlighted in red. $(b-d)$ Effects of Rldl on leaf polarity. (b) Mature Rld1 plant illustrating the tightly upward curled leaves. (c) Abaxial surface of a wild-type adult maize leaf, which comprises sheath (s) and blade (b) tissues separated by the auricle (a). (d) Abaxial surface of a partially adaxialized Rld1 leaf. Rld1 leaves frequently develop an ectopic abaxial ligule (black arrow) and clear sectors with fewer minor veins and no photosynthetic cell types (white arrow). (Reprinted, with permission, from Juarez et al. 2004b [CNature Publishing Group; http://www.nature.com].) covered gene regulatory mechanism in development and suggest that miRNAs may constitute a new class of developmental signaling molecules.

\section{miRNA BIOGENESIS AND FUNCTION IN GENE REGULATION}

miRNAs are endogenous small ( 22-mer) noncoding RNAs that mediate the cleavage or translational repression of target transcripts containing a complementary sequence. miRNAs were first identified in Caenorhabditis elegans (Lee et al. 1993; Reinhart et al. 2000), but have since been found in organisms as evolutionary distinct as plants and humans (Lagos-Quintana et al. 2001; Lau et al. 2001; Lee and Ambros 2001; Llave et al. 2002a; Park et al. 2002; Reinhart et al. 2002). To date, the cloning of small RNAs from Arabidopsis has lead to the identification of more than 40 distinct miRNAs that can be grouped into 22 miRNA families based on sequence similarity and target specificity (Jones-Rhoades and Bartel 2004). Most miRNAs are encoded by multigene families, such that the Arabidopsis genome includes 92 potential miRNA loci. However, this number is almost certainly an underestimate as additional miRNA families have been predicted using various computational approaches, but these await experimental verification (Bonnet et al. 2004; JonesRhoades and Bartel 2004). miRNAs are frequently conserved among distantly related animal species (Pasquinelli et al. 2000; see Bartel 2004). Similarly, 18 of the 22 Arabidopsis miRNA families have homologs, or predicted homologs, in rice (Reinhart et al. 2002; Bonnet et al. 2004; Jones-Rhoades and Bartel 2004; Wang et al. 2004). Such conservation suggests an important role for these miRNAs and the posttranscriptional regulation of their target genes.

miRNAs are initially transcribed as long primary transcripts called pri-miRNAs. These transcripts are processed into $~ 70-300$-nt stem-loop intermediates, known as the miRNA precursor or pre-miRNA. pre-miRNAs are subsequently cleaved to yield an imperfect duplex comprising the mature miRNA and the so-called miRNA*, which is derived from the opposite arm of the pre-miRNA stem. Production of the miRNA:miRNA* duplex from the pri-miRNA involves slightly different processes in animals and plants. In animals, the initial processing step is performed by the nuclear RNase III endonuclease, Drosha (Lee et al. 2003); whereas pre-miRNAs are cleaved in the cytoplasm by Dicer (Grishok et al. 2001; Hutvagner et al. 2001; Ketting et al. 2001), the RNase III enzyme that is also required for the production of siRNAs from double-stranded RNAs during RNAi (Bernstein et al. 2001). Drosha homologs are absent from plants, and both processing steps seem to be executed in the nucleus by the Dicer homolog, DICERLIKE1 (DCL1) (Park et al. 2002; Reinhart et al. 2002; Papp et al. 2003; Xie et al. 2003). Accordingly, pre-miRNAs do not accumulate to easily detectable levels in plants, as they do in animals.

The miRNA strand of the duplex becomes incorporated into a RNA-induced silencing complex (RISC), whereas the miRNA* gets degraded. The mechanism of 
RISC assembly is largely unknown, but is thought to involve a helicase activity that unwinds the miRNA: miRNA* duplex and selects, based on the thermodynamics of the duplex, the strand to enter the RISC (Khvorova et al. 2003; Schwarz et al. 2003). RISC loading in plants may also involve the double-stranded RNA (dsRNA) binding proteins HEN1 and HYPONASTIC LEAVES1 (HYL1), which affect the accumulation of miRNAs (Park et al. 2002; Han et al. 2004; Vazquez et al. 2004). Similar proteins from $C$. elegans and Drosophila facilitate the transfer of siRNAs to RISC (Tabara et al. 2002; Liu et al. 2003). Interestingly, both HYL1 and HEN1 are nuclear localized suggesting that, unlike in animal systems, miRNA production and RISC assembly in plants may occur entirely in the nucleus.

The miRNA-RISC complex can mediate mRNA cleavage or stall translation. The exact mechanism of repression is dictated in part by the level of complementarity between the miRNA and the target transcript (Hutvagner and Zamore 2002; Doench et al. 2003; Zeng et al. 2003). In animals, this complementarity is relatively limited and predominantly located within the $5^{\prime}$ end of the miRNA. As a result, most animal miRNAs act at the translational level. In contrast, plant miRNAs and their targets frequently possess near-perfect complementarity throughout. Most plant miRNA-mRNA interactions lead to cleavage of the target transcript at a position corresponding to nucleotides 10 and 11 of the miRNA (Llave et al. 2002b; Kasschau et al. 2003). However, the level of complementarity is not the sole factor that distinguishes between RNA cleavage and translational repression, at least not in plants. miR172 has near perfect complementarity to APETALA2 (AP2) but mainly affects AP2 translation (Aukerman and Sakai 2003; Chen 2004). The mode of miRNA action may also depend on the protein composition of RISC. ARGONAUTE (AGO) proteins form an integral component of all RISCs (Hammond et al. 2001; Mourelatos et al. 2002; Caudy et al. 2003), but most organisms encode multiple AGO family members that seem to mediate distinct RISC functions. For instance, the Arabidopsis zippy/ago 7 mutants display developmental phenotypes consistent with a defect in miRNA-mediated gene regulation, but AGO4 is required for transposon silencing, and AGO1 plays a role in both posttranscriptional and miRNA-mediated silencing (Fagard et al. 2000; Hunter et al. 2003; Zilberman et al. 2003; Vaucheret et al. 2004).

\section{miRNAs AS DEVELOPMENTAL SIGNALING MOLECULES}

The observation that the first plant miRNAs had nearperfect complementarity to their target mRNAs has enabled the identification of many target genes using computational approaches (Rhoades et al. 2002; Bonnet et al. 2004; Jones-Rhoades and Bartel 2004). The Arabidopsis miRNAs identified so far show a strong propensity to target transcription factor families or other genes controlling development. miRNAs that regulate other aspects of plant biology, such as basic metabolism and the response to particular pathogens or environmental stresses, have also been identified (Bonnet et al. 2004; Jones-Rhoades and Bartel 2004). Consistent with a role for miRNAs in a variety of developmental processes, mutations affecting their biogenesis are pleiotropic. For instance, dcll, hen 1, and hyll mutants exhibit overlapping defects in vegetative, floral, and reproductive development (Jacobsen et al. 1999; Chen et al. 2002; Han et al. 2004; Vazquez et al. 2004). Remarkably similar developmental defects are also observed in plants expressing viral suppressors of RNA silencing that interfere with miRNA function, such as P1/HC-Pro of Turnip mosaic virus, P19 of Tomato bushy stunt virus, or the P15 protein from Peanut clump virus (Kasschau et al. 2003; Dunoyer et al. 2004). However, not all mutations affecting miRNA production or function are as pleiotropic (e.g., zippy/ago 7 and hasty [Bollman et al. 2003; Hunter et al. 2003]), which could indicate partial redundancy or branching in the miRNA pathway. Interestingly, mutations in $A G O 1$ and the closely related PINHEAD/ZWILLE (PNH/ZLL) gene affect meristem function and adaxial/abaxial polarity (Bohmert et al. 1998; Moussian et al. 1998; Lynn et al. 1999; Kidner and Martienssen 2004), supporting a role for miRNA-mediated cleavage in the spatial regulation of hd-zipIII transcripts.

miRNA-directed cleavage products are relatively stable and numerous miRNA targets have been verified through detection of the predicted transcript fragments (see, e.g., Jones-Rhoades and Bartel 2004). Other targets have been confirmed by mutational analysis. These include the above-mentioned hd-zipIII family members, which are required for meristem function and adaxial/abaxial patterning (McConnell et al. 2001; Emery et al. 2003; Juarez et al. 2004b; McHale and Koning 2004; Zhong and Ye 2004). Dominant gain-of-function alleles of miR-JAW were identified in a collection of activation-tagged lines (Palatnik et al. 2003). miR-JAW regulates a subset of TCP genes required for the proper temporal transition from cell division to differentiation (Nath et al. 2003). This transition is delayed in the jaw-D mutants, which alters leaf shape. Plants expressing a miR-JAW-insensitive allele of TCP4 appear to have the opposite phenotype as they arrest early in development (Palatnik et al. 2003). Similar types of mutations also confirmed a role for miR164 in organ boundary formation by defining the expression domains of CUPSHAPED COTYLEDON1 (CUC1) and CUC2 (Laufs et al. 2004; Mallory et al. 2004) and for miR159 and miR172 in floral initiation and floral organ development. miR159 regulates the accumulation of AtMYB33, a component of the gibberellin response pathway and activator of the floral inducer LEAFY (Palatnik et al. 2003; Achard et al. 2004), whereas miR172 targets AP2 and several AP2-like transcription factors (Aukerman and Sakai 2003; Chen 2004). Interestingly, DCL1 and AGO1 are also targets for miRNA mediated posttranscriptional regulation, which suggests that miRNA biogenesis and function is controlled by a negative feedback mechanism (Xie et al. 2003; Vaucheret et al. 2004). 


\section{ADAXIAL/ABAXIAL AXIS SPECIFICATION BY miR166}

One possible reason why so many miRNAs target transcription factors or other regulators of plant development is that the active degradation of such transcripts may help facilitate changes in cell fate (Rhoades et al. 2002). For example, the down-regulation of $h d$-zipIII expression required for abaxial cell fate specification upon primordium emergence could be achieved by suppressing transcription of the hd-zipIII genes in cells on the abaxial side. However, abaxial-specific expression of miRNAs would enable a more rapid switch in cell fate by actively eliminating $h d$-zipIII transcripts inherited from the incipient primordium. Although this scenario is supported by characterization of the dominant hd-zipIII mutations, a comparative analysis of the hd-zipIII and miR165 or miR166 expression patterns at the cellular level would provide direct evidence that miRNA expression patterns can establish patterns of tissue organization during development. The relatively large size of the maize apex might simplify such a detailed comparison. We therefore took advantage of sequence conservation between the Arabidopsis and rice MIR166 genes and cloned fragments of several miR166 precursors from maize, which were used to determine the precise miR166 expression pattern by in situ hybridization (Juarez et al. 2004b).

miR165 and miR166 differ by a single C-U transition at position 17. Even though this nucleotide difference slightly decreases the complementarity of miR166 to the HD-ZIPIII transcripts, by substituting a G:C base pair with a G:U wobble, it does not affect the in vitro cleavage of PHV transcripts (Tang et al. 2003). Arabidopsis contains two MIR165 and seven MIR166 loci (Reinhart et al. 2002). Most pri-miRNA genes within a miRNA family show obvious sequence conservation in the miRNA*. Sequences outside this duplex usually exhibit little primary sequence similarity but maintain a propensity to form a dsRNA structure. Surprisingly, pair-wise comparisons between the MIR165 and MIR166 family members revealed extensive sequence conservation surrounding the predicted miRNA:miRNA* duplexes (Juarez et al. 2004b). The two MIR165 loci share six conserved domains, including the miRNA and miRNA* sequences, over an interval of $\sim 850$ nucleotides (nt). The pri-miR166 loci, MIR166a and MIR166b, share nine conserved sequence motifs over a region of $\sim 1.5 \mathrm{~kb}$. Similarly, MIR166c contains six sequence motifs that are conserved in MIR166d and four sequence motifs that are conserved in MIR166e. MIR166d also shows sequence homology to MIR166g in six domains over an $\sim 1 \mathrm{~kb}$ region. Most of these conserved sequence motifs are 30-50 nt in length, but some extend over 100 bases.

Because of the very efficient processing of pri-miRNAs into mature miRNAs, the MIR166 precursor transcripts are not detectable by northern blot analysis (Reinhart et al. 2002). However, primers derived from each of the nine conserved sequence motifs in MIR166a and MIR166b did allow the amplification of overlapping cDNA fragments from seedling RNA (T. Phelps-Durr and M. Timmermans, unpubl.). These conserved sequence motifs thus constitute part of the same pri-miRNA, which spans at least $1.4 \mathrm{~kb}$. Some pri-miRNA transcripts are processed by splicing (Aukerman and Sakai 2003), but these conserved sequences do not correspond to exons. They may, however, contain elements important for miRNA regulation or processing.

The rice genomic sequence contains at least six loci with the potential to produce miR166 homologs although MIR165 loci have not been identified (Reinhart et al. 2002). Like the Arabidopsis MIR 165 and MIR 166 family members, several of the potential MIR 166 loci from rice contain sequence homology outside the predicted miRNA and miRNA* sequences. OsMIR166c and $O s$ MIR $166 f$ share five regions of homology. OsMIR $166 \mathrm{~b}$ and $O s M I R 166 d$ share only three conserved sequence motifs and these include the miR166 and miRNA*. However, the sequence and arrangement of these three motifs is also conserved in Arabidopsis MIR166a and MIR166b (Juarez et al. 2004b). Irrespective of whether this third sequence motif is also part of the pri-miRNA in rice, its conservation between Arabidopsis and rice suggests it is likely conserved in maize as well.

Degenerate primers derived from these three conserved sequence motifs did indeed allow the amplification of several partial mir 166 cDNA clones from maize vegetative apex and inflorescence tissues (Juarez et al. 2004b). Six distinct reverse-transcriptase (RT)-dependent polymerase chain reaction (PCR) products were amplified from immature tassel RNA, but fewer mirl66 genes appear to be expressed in vegetative apices. Sequence analysis suggests at least four of the maize mirl66 genes (mir166a-mir166d) contain the three sequence motifs found to be conserved between Arabidopsis and rice. No other primary sequence similarities exist between these four maize genes and between the maize, rice, and Arabidopsis genes. Consistent with the amplification of fewer PCR products from vegetative apices, mirl66a and mir $166 \mathrm{~b}$ are expressed in both vegetative and inflorescence tissues, whereas expression of mirl66c and mir166d is limited to the inflorescence. As predicted by their regulatory role in development, miRNAs in both animals and plants accumulate in a temporal or tissue-specific pattern. However, these RT-PCR results suggest that individual pri-miRNA genes can display distinct tissue specificities. This could potentially be very significant, as this would reduce the level of redundancy among the primiRNA genes.

To examine directly whether miR166 defines the abaxial domain by restricting $h d$-zipIII expression to the adaxial side, we used in situ hybridization to compare the rldl and miR166 expression patterns at the cellular level (Juarez et al. 2004b). Approximately 60 rounds of amplification were required to detect mir $166 a$ pri-miRNA or pre-miRNA transcripts by RT-PCR. Accordingly, no hybridization signal was detected when vegetative apex sections were hybridized with a probe specific for the primiRNA. However, in situ hybridizations using a slightly larger fragment of mir166a that includes an antisense copy of miR166 revealed expression in developing leaf 
primordia (Fig. 4). miR166 accumulates on the abaxial side of the P1 leaf. In older leaf primordia, miR166 accumulates in a progressively broader domain extending laterally and adaxially. Only near the margins does miR166 expression remain limited to the abaxial side (Fig. 4a). rldl and miR166 thus exhibit complementary expression patterns in developing leaf primordia, consistent with a role for miR166 in defining the domain of rld1 expression. In contrast, the rldl expression domain in meristematic tissue is mainly controlled at the transcriptional level. rldl is expressed in the central zone of the SAM and in a stripe of cells that includes the incipient leaf primordium (Fig. 4b), whereas miR166 accumulates in a group of cells immediately below the incipient leaf opposite the P1 primordium. Interestingly, a gradient of weaker miR166 expression extends into the abaxial side of the P0 leaf (Fig. 4c) and a gradual decline in miR166 expression may also exist in older leaf primordia. As expected, rldl expression is ectopically expressed in the dominant Rld1 mutant and includes the miR166 expression domain below the incipient leaf and on the abaxial side of the P1 primordium (Fig. 4d), but rldl expression in the Rld1 SAM is unaffected. hd-zipIII genes also play a role in the adaxial/abaxial patterning of vascular bundles (Zhong and Ye 1999; Ratcliffe et al. 2000). rld1 and phb are expressed in provascular strands and expression becomes localized to adaxial pro-xylem cells when dis-

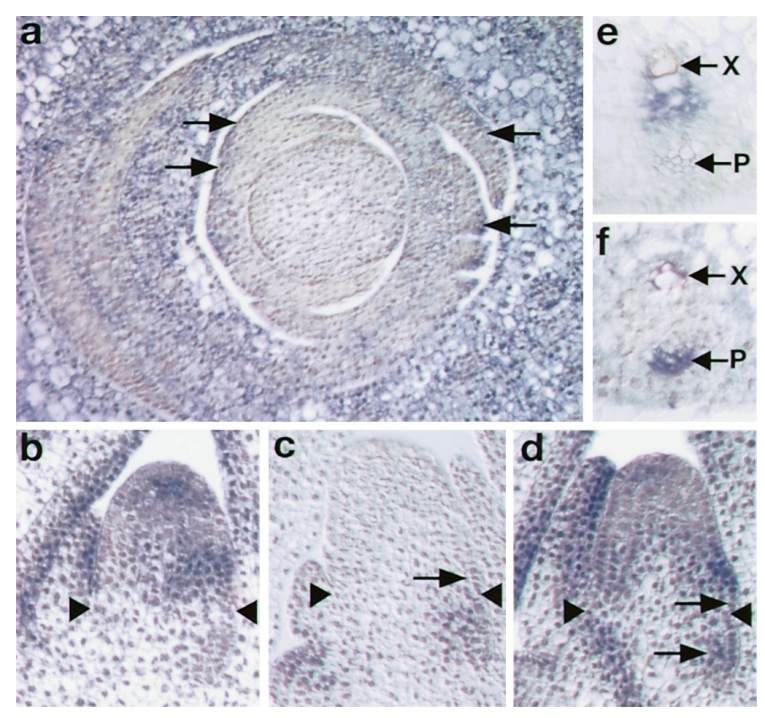

Figure 4. The hd-zipIII genes and miR166 have complementary expression patterns in developing leaf primordia. (a) Transverse section through a wild-type apex showing miR166 expression in leaf primordia but not in the SAM. Arrows mark regions with only abaxial miRNA166 expression. $(b, c)$ Longitudinal sections through wild-type apices showing $r l d l$ expression $(b)$ at the site of leaf initiation and miR166 expression $(c)$ on the abaxial side (arrow) and below the incipient leaf. Arrowheads mark the base of the incipient leaf. (d) Longitudinal sections through a Rldl apex showing rld 1 misexpression below and at the base of the incipient leaf (arrows). ( $e, f)$ Transverse sections through wildtype vascular bundles showing $p h b$ expression $(e)$ in adaxial proxylem cells and miR166 expression $(f)$ in abaxial phloem tissue. X, xylem; P, phloem. (Reprinted, with permission, from Juarez et al. 2004b [CNature Publishing Group; http://www.nature.com].) tinct phloem and xylem poles become apparent (Fig. 4e). At that time, miR166 accumulates in the abaxial phloem tissue (Fig. 4f).

\section{DISCUSSION}

Despite the differences in monocot and dicot leaf development, establishment of adaxial/abaxial polarity in both classes of angiosperms requires the spatial restriction of hd-zipIII expression to the adaxial side. In both Arabidopsis and maize this is mediated by the complementary, abaxial expression of miR166 and miR165 (Juarez et al. 2004b; Kidner and Martienssen 2004: T. Phelps-Durr and M. Timmermans, unpubl.). The miR165/166-directed cleavage of $h d$-zipIII transcripts is conserved even in the basal lineages of land plants and dates back more than 400 million years ago to the last common ancestor of the mosses and seed plants (Floyd and Bowman 2004). Conservation of let-7 across the animal phylogeny suggests a similar early origin for miRNA-mediated gene regulation in the metazoans (Pasquinelli et al. 2000). The differences in biogenesis, target gene complementarity, and usual mode of gene regulation between plant and animal miRNAs, together with the fact that no miRNA has thus far been identified that is conserved between animals and plants, suggest nonetheless that miRNAs may have arisen independently in each lineage (Bartel 2004). Alternatively, the regulation of genes by small RNAs may have arisen only once but the divergence of target genes may have lead to the fixation of distinct miRNA families in plants and animals.

The regulation of $h d$-zipIII gene expression by miR165/166 thus predates the origin of angiosperm leaves. Because hd-zipIII expression both on the adaxial side of lateral organs and in the adaxial pro-xylem cells is defined by the pattern of miR166 accumulation, these genes may have had an ancestral role in establishing polarity in vascular tissue of nonleafy plants, which was later co-opted in the adaxial/abaxial patterning of leaves (Juarez et al. 2004a). The role of the KAN genes in abaxial fate determination in developing leaves may similarly be derived from an ancestral function in vascular patterning (Emery et al. 2003). In Arabidopsis, KAN expression is limited to the abaxial or peripheral phloem cells, and mutational analysis suggests the KAN and $H D-Z I P I I I$ genes act antagonistically during both primordium and vascular development (Kerstetter et al. 2001; Emery et al. 2003).

Certain aspects of the pathway leading to adaxial/abaxial polarity have evolved between monocots and dicots. Maize yabby genes, in contrast to those of Arabidopsis, are expressed on the adaxial side of incipient and young leaf primordia, suggesting divergence in their regulation and function (Juarez et al. 2004a). Whereas the Arabidopsis HD-ZIPIII genes suppress YABBY expression (Siegfried et al. 1999; Eshed et al. 2001), yabby genes in maize are positively regulated by rld1. The maize and Arabidopsis yabby genes may share a role in mediating lateral outgrowth along the adaxial/abaxial boundary (Eshed et al. 2004; Juarez et al. 2004a). However, the Ara- 
bidopsis $Y A B B Y$ genes also specify abaxial fate, and this function is not conserved in maize (Sawa et al. 1999; Siegfried et al. 1999; Kumaran et al. 2002). Moreover, the miRNA loci involved in adaxial/abaxial patterning have diverged between Arabidopsis, rice, and maize. MIR165 loci have not been identified in the rice genome sequence (Reinhart et al. 2002), and no miR165 expression was observed in maize vegetative apices (T. Phelps-Durr and M. Timmermans, unpubl.). The number of MIR166 genes also varies between these species, and their sequences exhibit very limited primary sequence similarity outside the miR166 and miRNA*. Moreover, preliminary data suggest that the pattern of miR166 accumulation in Arabidopsis is not directly comparable to that in maize, although miR166 shows a dynamic pattern of expression throughout leaf development in both species (T. PhelpsDurr and M. Timmermans, unpubl.).

miR165/166 thus constitutes an important highly conserved polarizing signal. miR166 initially accumulates immediately below the incipient leaf but subsequently in a progressively broader domain including the adaxial side. This dynamic expression pattern and the gradient of miR166 expression into the incipient primordium is reminiscent of a movable signal, suggesting that expression of miR166 may be under such control just as expression of miR159 is regulated by the phytohormone gibberillin (Achard et al. 2004). Alternatively, miR166 may itself move between cells to set up a gradient of expression. This could imply a more active signaling role for miRNAs in development similar to that of peptide ligands or hormones. Although this hypothesis is currently unproven, the accumulation of miR166 in the phloem could be consistent with miR166 movement from the site of mir166 expression. Similarly suggestive, expression of a viral movement protein in the SAM that disrupts RNA trafficking causes formation of radial adaxialized leaves (Foster et al. 2002). The site of mir 166 expression is unknown but may coincide with the initial strong miR166 accumulation below the incipient primordium. If so, the mir 166 expression domain is likely established independently of abaxial determinants that function in the incipient primordium. mirl66 expression is also established independently of $l b l 1$ and miR166 acts upstream of rldl (M. Juarez and M. Timmermans, unpubl.).

Classical surgical experiments indicated that specification of adaxial cell fate requires a signal from the meristem (Sussex 1951, 1955). This signal could act via RLD1 and other HD-ZIPIII family members as they contain a START lipid-sterol binding domain. If so, RLD1 and other HD-ZIPIII proteins may specify adaxial/abaxial polarity in developing leaves by incorporating positional information established by two opposing signals that originate outside the incipient primordium: the adaxializing signal from the SAM and the miR166 signal from a potential signaling center below the incipient leaf (Fig. 5).

\section{ACKNOWLEDGMENTS}

The authors thank Catherine Kidner for helpful discussions and comments on the manuscript. We also thank Julie

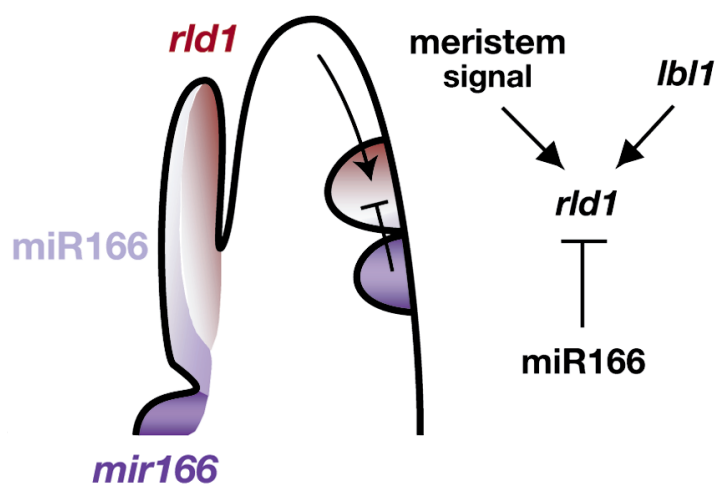

Figure 5. Adaxial/abaxial polarity in the maize leaf may be established by two distinct signals that originate outside the incipient leaf and act upon rldl. Expression of $r l d l$ in the incipient leaf depends on $l b l l$ and miR166. $l b l l$ positively affects the accumulation of rld 1 transcripts, whereas miR166 directs their cleavage. The miR166 signal initially accumulates immediately below the incipient leaf but gradually spreads into the abaxial domain, thus restricting rld 1 expression to the adaxial side. Specification of adaxial fate also requires a signal from the meristem, which may exert its effects via the START lipid-sterol binding domain of RLD1.

Thomas, Francois Bolduc, and Pawel Mazur for technical assistance; David Jackson for the SEM image of the maize SAM; and Tim Mulligan for plant care. This work was supported by grants from the NSF to M.C.P.T., and M.T.J. was in part funded by a W. Burghardt Turner fellowship.

\section{REFERENCES}

Achard P., Herr A., Baulcombe D.P., and Harberd N.P. 2004. Modulation of floral development by a gibberellin-regulated microRNA. Development 131: 3357.

Aukerman M.J. and Sakai H. 2003. Regulation of flowering time and floral organ identity by a microRNA and its APETALA2like target genes. Plant Cell 15: 2730.

Bartel D.P. 2004. MicroRNAs: Genomics, biogenesis, mechanism, and function. Cell 116: 281.

Bernstein E., Caudy A.A., Hammond S.M., and Hannon G.J. 2001. Role for a bidentate ribonuclease in the initiation step of RNA interference. Nature 409: 363.

Bohmert K., Camus I., Bellini C., Bouchez D., Caboche M., and Benning C. 1998. AGO1 defines a novel locus of Arabidopsis controlling leaf development. EMBOJ. 17: 170.

Bollman K.M., Aukerman M.J., Park M.Y., Hunter C., Berardini T.Z., and Poethig R.S. 2003. HASTY, the Arabidopsis ortholog of exportin 5/MSN5, regulates phase change and morphogenesis. Development 130: 1493.

Bonnet E., Wuyts J., Rouze P., and Van de Peer Y. 2004. Detection of 91 potential conserved plant microRNAs in Arabidopsis thaliana and Oryza sativa identifies important target genes. Proc. Natl. Acad. Sci. 101: 11511.

Caudy A.A., Ketting R.F., Hammond S.M., Denli A.M., Bathoorn A.M., Tops B.B., Silva J.M., Myers M.M., Hannon G.J., and Plasterk R.H. 2003. A micrococcal nuclease homologue in RNAi effector complexes. Nature 425: 411.

Chen X. 2004. A microRNA as a translational repressor of APETALA2 in Arabidopsis flower development. Science 303: 2022.

Chen X., Liu J., Cheng Y., and Jia D. 2002. HEN1 functions pleiotropically in Arabidopsis development and acts in C function in the flower. Development 129: 1085.

Doench J.G., Petersen C.P., and Sharp P.A. 2003. siRNAs can function as miRNAs. Genes Dev. 17: 438.

Dunoyer P., Lecellier C.H., Parizotto E.A., Himber C., and 
Voinnet O. 2004. Probing the microRNA and small interfering RNA pathways with virus-encoded suppressors of RNA silencing. Plant Cell 16: 1235.

Emery J.F., Floyd S.K., Alvarez J., Eshed Y., Hawker N.P., Izhaki A., Baum S.F., and Bowman J.L. 2003. Radial patterning of Arabidopsis shoots by class III HD-ZIP and KANADI Genes. Curr. Biol. 13: 1768.

Eshed Y., Baum S.F., Perea J.V., and Bowman J.L. 2001. Establishment of polarity in lateral organs of plants. Curr. Biol. 11: 1251

Eshed Y., Izhaki A., Baum S.F., Floyd S.K., and Bowman J.L. 2004. Asymmetric leaf development and blade expansion in Arabidopsis are mediated by KANADI and YABBY activities. Development 131: 2997.

Fagard M., Boutet S., Morel J.B., Bellini C., and Vaucheret H. 2000. AGO1, QDE-2, and RDE-1 are related proteins required for post-transcriptional gene silencing in plants, quelling in fungi, and RNA interference in animals. Proc. Natl. Acad. Sci. 97: 11650.

Floyd S.K. and Bowman J.L. 2004. Gene regulation: Ancient microRNA target sequences in plants. Nature 428: 485.

Foster T.M., Lough T.J., Emerson S.J., Lee R.H., Bowman J.L., Forster R.L., and Lucas W.J. 2002. A surveillance system regulates selective entry of RNA into the shoot apex. Plant Cell 14: 1497.

Grishok A., Pasquinelli A.E., Conte D., Li N., Parrish S., Ha I., Baillie D.L., Fire A., Ruvkun G., and Mello C.C. 2001. Genes and mechanisms related to RNA interference regulate expression of the small temporal RNAs that control C. elegans developmental timing. Cell 106: 23.

Hammond S.M., Boettcher S., Caudy A.A., Kobayashi R., and Hannon G.J. 2001. Argonaute2, a link between genetic and biochemical analyses of RNAi. Science 293: 1146.

Han M.H., Goud S., Song L., and Fedoroff N. 2004. The Arabidopsis double-stranded RNA-binding protein HYL1 plays a role in microRNA-mediated gene regulation. Proc. Natl. Acad. Sci. 101: 1093.

Hunter C., Sun H., and Poethig R.S. 2003. The Arabidopsis heterochronic gene ZIPPY is an ARGONAUTE family member. Curr. Biol. 13: 1734.

Hutvagner G. and Zamore P.D. 2002. A microRNA in a multiple-turnover RNAi enzyme complex. Science 297: 2056.

Hutvagner G., McLachlan J., Pasquinelli A.E., Balint E., Tuschl T., and Zamore P.D. 2001. A cellular function for the RNAinterference enzyme Dicer in the maturation of the let-7 small temporal RNA. Science 293: 834.

Jacobsen S.E., Running M.P., and Meyerowitz E.M. 1999. Disruption of an RNA helicase/RNAse III gene in Arabidopsis causes unregulated cell division in floral meristems. Development 126: 5231.

Jones-Rhoades M.W. and Bartel D.P. 2004. Computational identification of plant microRNAs and their targets, including a stress-induced miRNA. Mol. Cell 14: 787.

Juarez M.T., Twigg R.W., and Timmermans M.C.P. 2004a. Specification of adaxial cell fate during maize leaf development. Development 131: 4533.

Juarez M.T., Kui J.S., Thomas J., Heller B.A., and Timmermans M.C.P. 2004b. microRNA-mediated repression of rolled leaf1 specifies maize leaf polarity. Nature 428: 84.

Kasschau K.D., Xie Z., Allen E., Llave C., Chapman E.J., Krizan K.A., and Carrington J.C. 2003. P1/HC-Pro, a viral suppressor of RNA silencing, interferes with Arabidopsis development and miRNA function. Dev. Cell 4: 205.

Kerstetter R.A., Bollman K., Taylor R.A., Bomblies K., and Poethig R.S. 2001. KANADI regulates organ polarity in Arabidopsis. Nature 411: 706.

Ketting R.F., Fischer S.E., Bernstein E., Sijen T., Hannon G.J., and Plasterk R.H. 2001. Dicer functions in RNA interference and in synthesis of small RNA involved in developmental timing in C. elegans. Genes Dev. 15: 2654.

Khvorova A., Reynolds A., and Jayasena S.D. 2003. Functional siRNAs and miRNAs exhibit strand bias. Cell 115: 209.

Kidner C.A. and Martienssen R.A. 2004. Spatially restricted microRNA directs leaf polarity through ARGONAUTE1. Nature
428: 81

Kumaran M.K., Bowman J.L., and Sundaresan V. 2002. YABBY polarity genes mediate the repression of $K N O X$ homeobox genes in Arabidopsis. Plant Cell 14: 2761

Lagos-Quintana M., Rauhut R., Lendeckel W., and Tuschl T. 2001. Identification of novel genes coding for small expressed RNAs. Science 294: 853.

Lau N.C., Lim L.P., Weinstein E.G., and Bartel D.P. 2001. An abundant class of tiny RNAs with probable regulatory roles in Caenorhabditis elegans. Science 294: 858.

Laufs P., Peaucelle A., Morin H., and Traas J. 2004. MicroRNA regulation of the $C U C$ genes is required for boundary size control in Arabidopsis meristems. Development 131: 4311.

Lee R.C. and Ambros V. 2001. An extensive class of small RNAs in Caenorhabditis elegans. Science 294: 862.

Lee R.C., Feinbaum R.L., and Ambros V. 1993. The C. elegans heterochronic gene lin-4 encodes small RNAs with antisense complementarity to lin-14. Cell 75: 843.

Lee Y., Ahn C., Han J., Choi H., Kim J., Yim J., Lee J., Provost P., Radmark O., Kim S., and Kim V.N. 2003. The nuclear RNase III Drosha initiates microRNA processing. Nature 425: 415 .

Liu Q., Rand T.A., Kalidas S., Du F., Kim H.E., Smith D.P., and Wang X. 2003. R2D2, a bridge between the initiation and effector steps of the Drosophila RNAi pathway. Science 301: 1921.

Llave C., Kasschau K.D., Rector M.A., and Carrington J.C. 2002a. Endogenous and silencing-associated small RNAs in plants. Plant Cell 14: 1605.

Llave C., Xie Z., Kasschau K.D., and Carrington J.C. 2002 b. Cleavage of Scarecrow-like mRNA targets directed by a class of Arabidopsis miRNA. Science 297: 2053.

Lynn K., Fernandez A., Aida M., Sedbrook J., Tasaka M., Masson P., and Barton M.K. 1999. The PINHEAD/ZWILLE gene acts pleiotropically in Arabidopsis development and has overlapping functions with the ARGONAUTE1 gene. Development 126: 469.

Mallory A.C., Dugas D.V., Bartel D.P., and Bartel B. 2004. MicroRNA regulation of NAC-domain targets is required for proper formation and separation of adjacent embryonic, vegetative, and floral organs. Curr. Biol. 14: 1035.

McConnell J.R., Emery J., Eshed Y., Bao N., Bowman J., and Barton M.K. 2001. Role of PHABULOSA and PHAVOLUTA in determining radial patterning in shoots. Nature 411: 709.

McHale N.A. and Koning R.E. 2004. MicroRNA-directed cleavage of Nicotiana sylvestris PHAVOLUTA mRNA regulates the vascular cambium and structure of the apical meristem. Plant Cell 16: 1730.

Mourelatos Z., Dostie J., Paushkin S., Sharma A., Charroux B., Abel L., Rappsilber J., Mann M., and Dreyfuss G. 2002. miRNPs: A novel class of ribonucleoproteins containing numerous microRNAs. Genes Dev. 16: 720.

Moussian B., Schoof H., Haecker A., Jurgens G., and Laux T. 1998. Role of the ZWILLE gene in the regulation of central shoot meristem cell fate during Arabidopsis embryogenesis. EMBO J. 17: 1799 .

Nath U., Crawford B.C., Carpenter R., and Coen E. 2003. Genetic control of surface curvature. Science 299: 1404.

Nelson J.M., Lane B., and Freeling M. 2002. Expression of a mutant maize gene in the ventral leaf epidermis is sufficient to signal a switch of the leaf's dorsoventral axis. Development 129: 4581.

Otsuga D., DeGuzman B., Prigge M.J., Drews G.N., and Clark S.E. 2001. REVOLUTA regulates meristem initiation at lateral positions. Plant J. 25: 223.

Palatnik J.F., Allen E., Wu X., Schommer C., Schwab R., Carrington J.C., and Weigel D. 2003. Control of leaf morphogenesis by microRNAs. Nature 425: 257.

Papp I., Mette M.F., Aufsatz W., Daxinger L., Schauer S.E., Ray A., van der Winden J., Matzke M., and Matzke A.J. 2003. Evidence for nuclear processing of plant micro RNA and short interfering RNA precursors. Plant Physiol. 132: 1382.

Park W., Li J., Song R., Messing J., and Chen X. 2002. CARPEL FACTORY, a Dicer homolog, and HEN1, a novel protein, act 
in microRNA metabolism in Arabidopsis thaliana. Curr. Biol. 12: 1484.

Pasquinelli A.E., Reinhart B.J., Slack F., Martindale M.Q., Kuroda M.I., Maller B., Hayward D.C., Ball E.E., Degnan B., Muller P., Spring J., Srinivasan A., Fishman M., Finnerty J., Corbo J., Levine M., Leahy P., Davidson E., and Ruvkun G. 2000. Conservation of the sequence and temporal expression of let-7 heterochronic regulatory RNA. Nature 408: 86 .

Ratcliffe O.J., Riechmann J.L., and Zhang J.Z. 2000. INTERFASCICULAR FIBERLESS1 is the same gene as REVOLUTA. Plant Cell 12: 315.

Reinhart B.J., Weinstein E.G., Rhoades M.W., Bartel B., and Bartel D.P. 2002. MicroRNAs in plants. Genes Dev. 16: 1616.

Reinhart B.J., Slack F.J., Basson M., Pasquinelli A.E., Bettinger J.C., Rougvie A.E., Horvitz H.R., and Ruvkun G. 2000. The 21-nucleotide let-7 RNA regulates developmental timing in Caenorhabditis elegans. Nature 403: 901.

Rhoades M.W., Reinhart B.J., Lim L.P., Burge C.B., Bartel B., and Bartel D.P. 2002. Prediction of plant microRNA targets. Cell 110: 513

Sawa S., Watanabe K., Goto K., Liu Y.G., Shibata D., Kanaya E., Morita E.H., and Okada K. 1999. FILAMENTOUS FLOWER, a meristem and organ identity gene of Arabidopsis, encodes a protein with a zinc finger and HMG-related domains. Genes Dev. 13: 1079.

Schwarz D.S., Hutvagner G., Du T., Xu Z., Aronin N., and Zamore P.D. 2003. Asymmetry in the assembly of the RNAi enzyme complex. Cell 115: 199.

Siegfried K.R., Eshed Y., Baum S.F., Otsuga D., Drews G.N., and Bowman J.L. 1999. Members of the YABBY gene family specify abaxial cell fate in Arabidopsis. Development 126: 4117.

Sussex I.M. 1951. Experiments on the cause of dorsiventrality in leaves. Nature 167: 651 .

1955. Morphogenesis in Solanum tuberosum L.: Experimental investigation of leaf dorsiventrality and orientation in the juvenile shoot. Phytomorphology 5: 286.

Tabara H., Yigit E., Siomi H., and Mello C.C. 2002. The dsRNA binding protein RDE-4 interacts with RDE-1, DCR-1, and a
DExH-box helicase to direct RNAi in C. elegans. Cell 109: 861.

Tang G., Reinhart B.J., Bartel D.P., and Zamore P.D. 2003. A biochemical framework for RNA silencing in plants. Genes Dev. 17: 49.

Timmermans M.C.P., Schultes N.P., Jankovsky J.P., and Nelson T. 1998. Leafbladeless 1 is required for dorsoventrality of lateral organs in maize. Development 125: 2813.

Vaucheret H., Vazquez F., Crete P., and Bartel D.P. 2004. The action of ARGONAUTE1 in the miRNA pathway and its regulation by the miRNA pathway are crucial for plant development. Genes Dev. 18: 1187.

Vazquez F., Gasciolli V., Crete P., and Vaucheret H. 2004. The nuclear dsRNA binding protein HYL1 is required for microRNA accumulation and plant development, but not posttranscriptional transgene silencing. Curr. Biol. 14: 346.

Waites R. and Hudson A. 1995. phantastica: A gene required for dorsoventrality of leaves in Antirrhinum majus. Development 121: 2143 .

Wang J.F., Zhou H., Chen Y.Q., Luo Q.J., and Qu L.H. 2004. Identification of 20 microRNAs from Oryza sativa. Nucleic Acids Res. 32: 1688.

Wardlaw C.W. 1949. Experiments on organogenesis in ferns. Growth (suppl.) 13: 93.

Xie Z., Kasschau K.D., and Carrington J.C. 2003. Negative feedback regulation of DICER-LIKE1 in Arabidopsis by microRNA-guided mRNA degradation. Curr. Biol. 13: 784.

Zeng Y., Yi R., and Cullen B.R. 2003. MicroRNAs and small interfering RNAs can inhibit mRNA expression by similar mechanisms. Proc. Natl. Acad. Sci. 100: 9779.

Zhong R. and Ye Z.H. 1999. IFL1, a gene regulating interfascicular fiber differentiation in Arabidopsis, encodes a homeodomain-leucine zipper protein. Plant Cell 11: 2139.

. 2004. Amphivasal vascular bundle1, a gain-of-function mutation of the IFL1/REV gene, is associated with alterations in the polarity of leaves, stems and carpels. Plant Cell Physiol. 45: 369.

Zilberman D., Cao X., and Jacobsen S.E. 2003. ARGONAUTE4 control of locus-specific siRNA accumulation and DNA and histone methylation. Science 299: 716. 


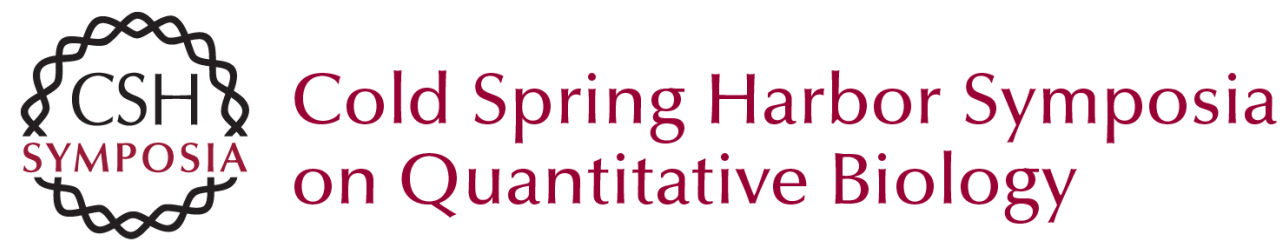

\section{A Conserved microRNA Signal Specifies Leaf Polarity}

M.C.P. TIMMERMANS, M.T. JUAREZ and T.L. PHELPS-DURR

Cold Spring Harb Symp Quant Biol 2004 69: 409-418

Access the most recent version at doi:10.1101/sqb.2004.69.409

References This article cites 78 articles, 45 of which can be accessed free at: http://symposium.cshlp.org/content/69/409.full.html\#ref-list-1

\section{License}

Email Alerting Receive free email alerts when new articles cite this article - sign up in Service the box at the top right corner of the article or click here. 